\title{
Un chatbot privado y seguro en Moodle para facilitar procesos de enseñanza-aprendizaje y administración \\ A private and secure chatbot in Moodle to facilitate teaching-learning and administration processes
}

\author{
Daniel Amo ${ }^{1}$, David Fonseca ${ }^{2}$, Bernat Rovirosa ${ }^{1}$, Xavi Canaleta $^{1}$, Eduard de Torres Gómez ${ }^{1}$, Joan Navarro ${ }^{1}$, Xavier \\ Solé \\ email Autor1, email Autor2, email Autor3 \\ ${ }^{1}$ Departament d'Enginyeria \\ La Salle, Universitat Ramon llull \\ Barcelona, España \\ ${ }^{2}$ Departament d'Arquitectura \\ La Salle, Universitat Ramon Llull \\ Barcelona, España
}

\begin{abstract}
Resumen- Los chatbots son programas auxiliares que ayudan a navegar por los distintos menús de las soluciones informáticas, encontrar información, solucionar dudas o poner en contacto con la persona que pueda resolver las no consideradas en su algoritmo. Integrados en un entorno virtual de aprendizaje presentan oportunidades y retos. Por un lado, se les suman a las funcionalidades citadas otras nuevas como dar soporte al aprendizaje de los estudiantes mediante la localización de tareas pendientes, agilizar la gestión académica de los docentes como consultar alumnos en riesgo de abandono, o facilitar la administración de la plataforma como activado o desactivado rápido de configuraciones usando el chatbot como una línea de comandos. Por otro lado, estas soluciones se ejecutan en servidores de terceros, generándose graves problemas de privacidad y seguridad en cuanto a datos personales. En este trabajo se propone un chatbot en forma de conector básico para Moodle el cual ahora (1) permite resolver problemas clásicos como el "listado de la muerte" y limitaciones en el buscador, (2) se ejecute en local para proteger y asegurar niveles éticos y legales de la privacidad, identidad, seguridad y confidencialidad de datos, datos personales y metadatos, (3) registre interacciones, y (4) sea de código abierto.
\end{abstract}

Palabras clave: chatbot, moodle, elearning, privacidad de datos, seguridad, identidad digital, analítica del aprendizaje.

Abstract- Chatbots are auxiliary programs that help navigate through the different menus of computer solutions, find information, solve doubts, or put in contact with the person who can solve those not considered in their algorithm. Integrated into a virtual learning environment they present both opportunities and problems. On the one hand, new functionalities are added to the aforementioned, such as supporting student learning by locating pending tasks, streamlining the academic management of teachers such as consulting students at risk of dropping out, or facilitating the administration of the platform, as a quick enabler or disabler of settings using the chatbot as a command line. On the other hand, these solutions are run on third-party servers, generating serious privacy and security problems in terms of personal data. In this work, a chatbot is proposed in the form of a plugin for Moodle which, for now, (1) allows solving classic problems such as the "scroll of death" and limitations in the search engine, (2) runs locally to protect and ensure ethical standards and legal privacy, identity, security and confidentiality of data, personal data and metadata, (3) record interactions, and (4) is open source.

Keywords: chatbot, moodle, elearning, data privacy, security, digital identity, learning analytics.

\section{INTRODUCCIÓN}

En los Entornos Virtuales de Aprendizaje (EVA) es habitual encontrar un seguido de contenidos y actividades (recursos) que en grandes cantidades pueden suponer un problema. En Moodle se califica como "el listado de la muerte" (Ballantyne, 2017; Duque, 2017; Lande, 2016) ("scroll of death" en inglés), aquel curso que tiene muchos recursos por lo que se hace arduo encontrar aquello deseado. Por este motivo los EVA incorporan un buscador cuya funcionalidad consiste en localizar información y evitar el caos entre muchos recursos. No obstante, incluso con las opciones avanzadas de búsqueda, en caso de disponerlas, a menudo los resultados no son concluyentes o muestran incluso demasiada información. Estas limitaciones vienen dadas por la no disposición de una inteligencia artificial que aumente sus posibilidades. Corregir estas imprecisiones solo es posible recodificando el buscador y resolviendo problemáticas ad hoc.

En otro orden de soluciones de búsqueda se encuentran los chatbots (Ranoliya, Raghuwanshi, \& Singh, 2017). Los chatbots son programas auxiliares que a modo de asistentes ayudan a navegar por los distintos menús de las soluciones informáticas, encontrar información, solucionar dudas o poner en contacto con la persona que pueda resolver las no consideradas en su algoritmo.

Los chatbots integrados en un EVA presentan tanto oportunidades como retos, tales como soporte individualizado (Clarizia, Colace, Lombardi, Pascale, \& Santaniello, 2018) o personalización del aprendizaje (Hien, Cuong, Nam, Nhung, \& Thang, 2018). Por un lado, pueden resolver de forma eficaz la 
búsqueda de contenidos en un EVA y se le pueden sumar a las funcionalidades citadas otras nuevas, entre otras:

- Mejorar el soporte al aprendizaje de los estudiantes mediante la localización de tareas pendientes, recursos de aprendizaje, cursos virtuales, contactar con el soporte técnico de la institución o incluso tramitar el envío de una tarea.

- Agilizar la gestión académica de los docentes como consultar alumnos en riesgo de abandono, mostrar estadísticas avanzadas, calificar tareas entregadas o hasta crear recursos y actividades en un curso.

- Facilitar la administración de la plataforma como activar o desactivar configuraciones, crear cursos o incluso gestionar usuarios.

- Convertir los chatbots como una línea de comandos aporta nuevas formas inimaginables de interactuar con y gestionar los EVA. Conversar con un chatbot da la posibilidad de tener ayuda asistida en la ejecución de un comando, búsqueda de información o realización de cualquiera de las funcionalidades mencionadas. Disponer de un chatbot a modo de interfaz de comandos agiliza la ejecución de las funcionalidades para aquellos usuarios que las conozcan, sepan cómo utilizar los comandos y no requieran asistencia.

Por otro lado, estas soluciones se ejecutan en plataformas de terceros, pudiéndose generar graves problemas de privacidad y seguridad en cuanto a datos personales (D. Amo, 2020; Daniel Amo, Alier, García-Peñalvo, Fonseca, \& Casañ, 2020). En un chatbot, conversacional o de comandos, pueden introducirse datos personales y datos educativos sensibles. Cuando estos datos son enviados a servidores de terceros las instituciones educativas pierden el control de los datos dando lugar a un posible estado de desconfianza y problemas de fuga de datos, malos usos o incluso transferencia a terceros no autorizados.

En concreto, para solucionar los problemas citados y hacer disponibles las oportunidades citadas, se propone un chatbot que se ejecuta en el mismo servidor que la instancia de Moodle usando las mismas tecnologías de scripts de servidor y de bases de datos para una absoluta compatibilidad, al mismo tiempo que se facilita la instalación y gestión a los administradores mediante el desarrollo de un conector.

Este trabajo se organiza en 5 secciones. La primera sirve de introducción. La segunda introduce el contexto del estudio. La tercera presenta la metodología aplicada. La cuarta muestra los resultados de la ejecución metodológica. Como última sección, la quinta muestra las conclusiones del estudio.

\section{CONTEXTO}

El estudio se enmarca en un EVA como es Moodle, una de las plataformas de esta categoría más utilizadas en el territorio español, siendo además este territorio el que tiene más instalaciones en relación al resto del mundo (Moodle, 2021). La necesidad de la integración de un chatbot con Moodle viene dada por las limitaciones que ofrece el buscador integrado, considerando relevante la problemática de búsqueda de contenido en aquellos cursos de Moodle con contenido considerable.
El objetivo principal del trabajo es ofrecer un método de búsqueda de contenido en Moodle de acceso inmediato e interactivo, que resuelva las limitaciones del buscador de serie, que asegure privacidad y seguridad en el proceso, y permita añadir o ejecutarse como interfaz de nuevas funcionalidades que agilicen la administración y procesos de enseñanzaaprendizaje.

En este trabajo, se muestra y se hace disponible el desarrollo de un chatbot que:

- Evite la exposición de datos educativos asegurando un nivel adecuado de la privacidad según los establecido en el Reglamento General de Protección de Datos (EP and the CEU, 2016).

- Permita la búsqueda y resuelva las limitaciones del buscador global de contenidos sin salir del contexto de Moodle.

- Esté preparado para incorporar las funcionalidades citadas arriba como segunda fase del trabajo.

\section{DESCRIPCIÓN}

La aproximación metodológica de este trabajo se basa en un diseño multimétodo consistente en las siguientes fases:

- Revisión del estado del arte en cuanto a chatbots y Moodle.

- Elaboración de un diseño que respete la privacidad y seguridad de los datos de roles educativos.

- Desarrollo del conector en Moodle bajo requisitos iniciales.

- Publicación del código en abierto y presentación en la MoodleMoot como solución funcional.

\section{A. Revisión del estado del arte}

Existe literatura relacionada con chatbots y Moodle donde los autores exponen distintas soluciones. Dehon et al. (Dehon et al., 2018) desarrollan una solución basada en la API de Facebook a la que llaman CVChatbot y consiste en enviar a los estudiantes notificaciones vía Facebook Messenger de eventos interesantes de Moodle. Oliveira et al. (Oliveira, Espindola, Barwaldt, Ribeiro, \& Pias, 2019) desarrollan un chatbot para Moodle utilizando dos APIs de servicios de terceros, Facebook e IBM Watson, para comunicar Moodle con Facebook Messenger como interfaz del chatbot y los servicios de IBM Watson para análisis del texto introducido. Wijaya y Pebriantara (Wijaya \& Pebriantara, 2018) exponen su manera de integrar un chatbot en Moodle mediante una solución basada en la API de Dialogflow de Google que se ofrece como "una plataforma de comprensión del lenguaje natural utilizada para diseñar e integrar una interfaz de usuario conversacional en aplicaciones móviles, aplicaciones web, dispositivos, bots, sistemas de respuesta de voz interactivos y usos relacionados”.

Otras soluciones integrables en Moodle, como la propuesta por Lee et al. (Lee et al., 2020), muestran una integración de cuatro servicios de terceros para poder activar la comunicación con estudiantes en las plataformas Telegram, Facebook y Line mediante el uso de Dialogflow y almacén de datos en Firebase, estos dos últimos servicios de Google.

A pesar de este panorama sensible en cuanto a datos educativos encontramos los autores Souali et al. (Souali, Rahmaoui, Ouzzif, \& El Haddioui, 2019) que proponen una 


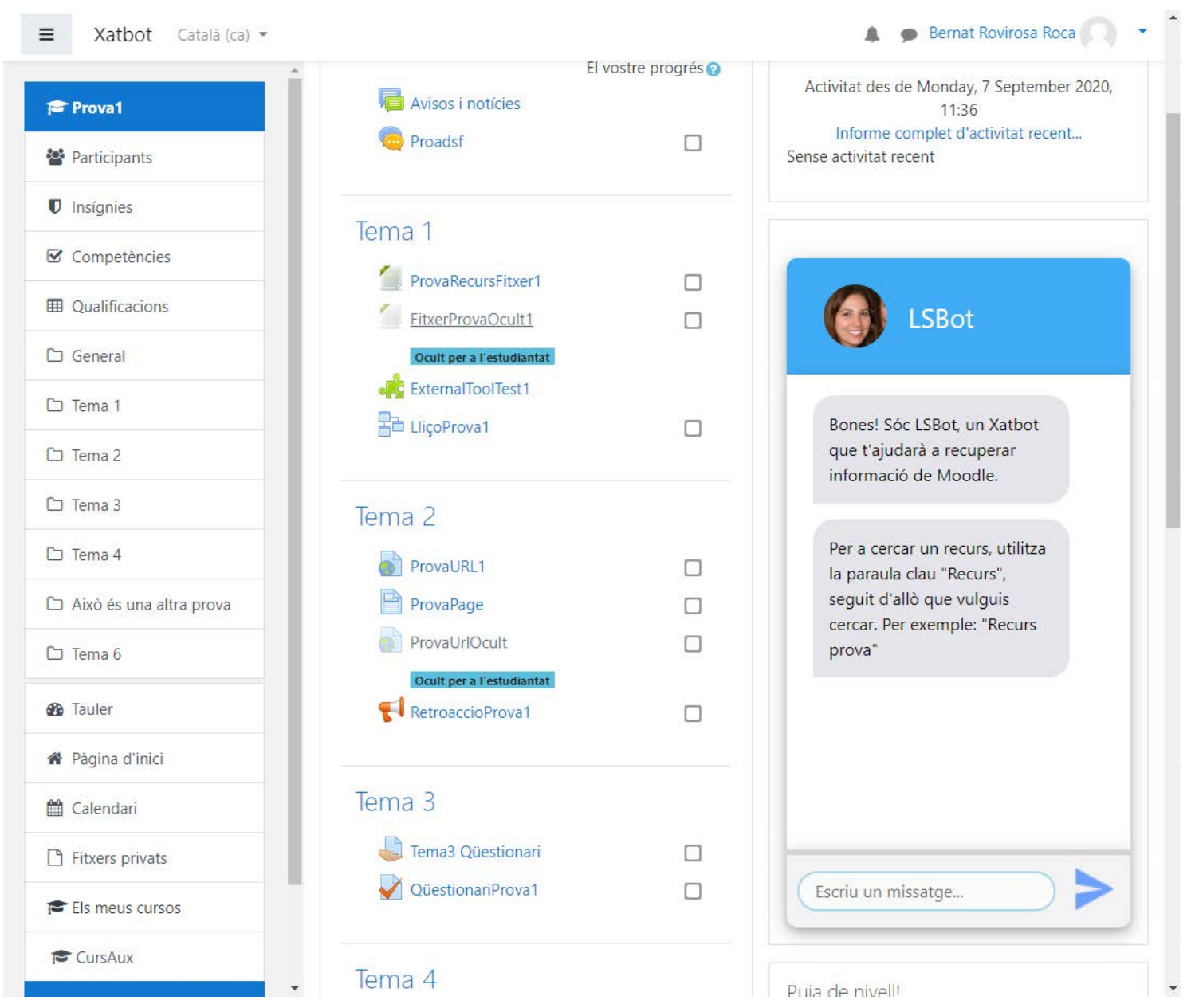

Figura 2: Interfaz del chatbot integrada con el mismo estilo en la interfaz de Moodle.

arquitectura chatbot para Moodle de desarrollo propio en PHP y con una base de datos adicional para realizar futuros análisis. No obstante, en búsquedas específicas de publicaciones de estos autores en años recientes no muestran ni la interfaz ni la integración con Moodle en código abierto.

Tras contactar con Moodle y dos de sus distribuidores oficiales, se confirma que en el momento de la escritura de este trabajo no hay una solución chatbot disponible con las características propuestas. Se remite a algunas entidades como SpringML (SpringML, 2019) basado en Dialogflow y un distribuidor oficial australiano que en el encuentro Moodlemoot del 2017 presentó una prueba de concepto y en desarrollo basado en Amazon Web Services (Catalyst IT, 2017).

El estado del arte confirma la necesidad de desarrollar un chatbot funcional para Moodle y que se ejecute en local para prevenir malos usos en la transferencia de datos educativos, como podrían ser los académicos o personales identificativos.

\section{B. Tecnología}

Para resolver posibles problemas relacionados con datos y aprovechar los beneficios de un chatbot, se ha desarrollado un chatbot como conector de Moodle considerando las siguientes premisas requisito:

- Utilizar tecnologías nativas de Moodle.

- Implementar una plataforma chatbot de ejecución en local (D. Amo et al., 2020).
- Emplear tecnologías y plataformas de código abierto.

- Implementar considerando requisitos no funcionales como un fácil mantenimiento, gestión y eficiencia

- Compartir código fuente en abierto AUTOREF y a la comunidad Moodle AUTOREF.

Por lo tanto, es objetivo dentro del desarrollo del conector generar compatibilidad tecnológica con el sistema Moodle. Se facilita de esta manera cuestiones de gestión e incluso de seguridad de datos, a la vez que el sistema sea fácil de escalar o de añadir funcionalidades nuevas ad hoc a las instituciones que lo integren, por ejemplo, las funcionalidades adicionales citadas en la introducción.

\section{Resultados}

El resultado del desarrollo, y como objetivos del trabajo, 1) se pone a disposición en código abierto (Rovirosa \& Amo, 2020); 2) asimismo, se envía para aprobación a la MoodleMoot España 2020, siendo aceptado por interés de los usuarios Moodle, y presentándose en noviembre del mismo 2020 (La Salle, 2020).

Se presentan los resultados obtenidos en cuanto a arquitectura, analítica del aprendizaje e interfaz del conector.

\section{A. Arquitectura}




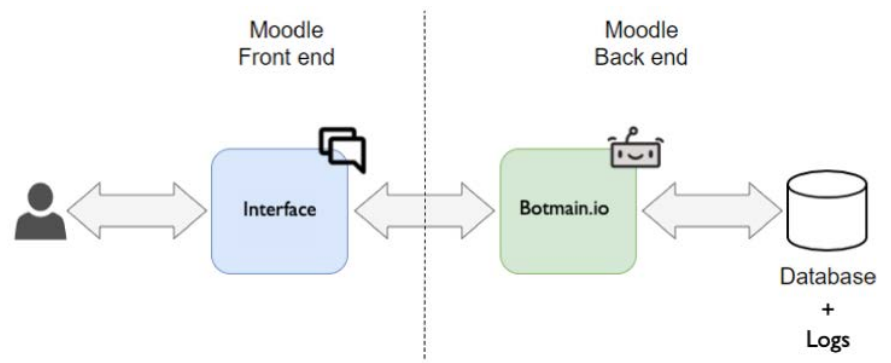

Figura 1: Arquitectura del chatbot desarrollado para Moodle en base a la plataforma de código abierto https://botman.io/.

Considerando los requisitos, se desarrolla el chatbot con lenguaje PHP y base de datos MySQL, dos tecnologías nativas de Moodle, utilizando la plataforma BotMan (Pociot, 2017).

BotMan es un marco de desarrollo de chatbots que facilita la implementación ad hoc de un chatbot dentro de los servidores de la institución educativa. El lenguaje de programación de BotMan es el PHP y su estructura permite conectarlo a cualquier base de datos, MySQL en el caso de Moodle. Se observa en la Figura 1 el diseño de la arquitectura del chatbot desarrollado. El código del chatbot reside en el core de Moodle como un conector bloque. De esta manera se integra en Moodle y el usuario no debe utilizar una plataforma externa con modo de funcionamiento distinto. La conexión del chatbot se realiza a la misma base de datos Moodle donde residen las tablas asociadas bajo los requisitos de privacidad y seguridad de la institución.

\section{B. Interfaz del contector}

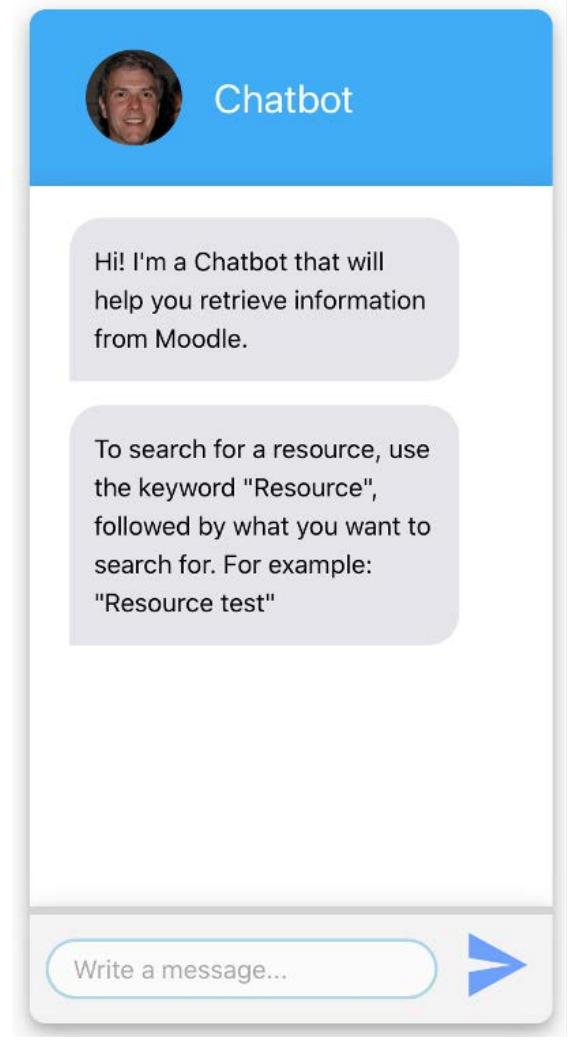

Figura 3: Mensaje de bienvenida del chatbot configurado en inglés y con una cara humana.
La Figura 2 muestra la integración del conector en la interfaz visual de Moodle con el mensaje de bienvenida y listo para recibir instrucciones.

La Figuras 3 muestra con más detalle el mensaje de bienvenida, demostrando que es posible configurar la interfaz cambiando el idioma al inglés y la imagen que da cara humana al asistente.

La Figura 4 muestra una interacción con el chatbot en el que se le pide cualquier recurso con cualquier nombre dando opción el chatbot de escoger el tipo de recurso siendo fichero, dirección web, tarea o incluso cualquiera.

\section{Analítica del aprendizaje}

El sistema genera trazas de uso para posteriores análisis de rendimiento, uso y perfeccionamiento. Implementar un sistema de registros propio del conector implica tanto la creación como gestión de nuevas tablas y una manera de visualizar las trazas en el mismo Moodle. Aunque un sistema propio permite más control sobre los datos que se puedan registrar, se decide usar el sistema que proporciona Moodle, puesto que está preparado para añadir eventos nuevos (trazas) $\mathrm{y}$, aunque no es tan completo como un personalizado, almacena suficientes datos como para realizar un análisis general del uso y funcionamiento del chatbot.

La seguridad de Moodle que ofrece por defecto en cuanto a acceso a estas trazas es suficientemente robusta. Los roles que pueden ver todos los logs de Moodle son el de administrador del sitio y el de gestor; el rol de profesor solo puede ver lo que ha sucedido dentro de su curso.

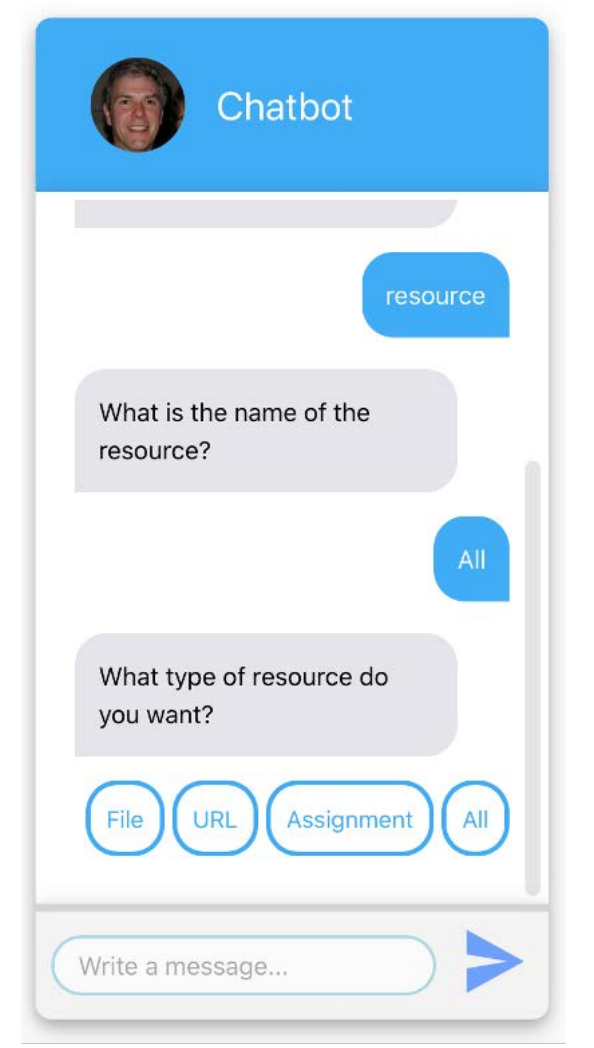

Figura 4: Muestra de interacción con el chatbot. 
En esta primera versión del conector se utiliza el sistema de $\log s$ de Moodle, puesto que permite un despliegue rápido, una interfaz de visualización conocida por el usuario, ver quién utiliza el conector, el número de peticiones que recibe y desde donde se utiliza, además de registrar los siguientes datos:

- El contexto donde se utiliza el conector (curso, recurso...).

- La acción de lectura.

- El nivel educativo considerado como LEVEL_OTHER.

- La descripción "'User with id X has searched a resource using the chatbot", donde $X$ es el identificador de usuario de Moodle.

\section{CONCLUSIONES}

Se demuestra en el trabajo que es posible desarrollar un chatbot de código abierto para instituciones educativas cuya ejecución se realice en el mismo servidor donde reside el EVA atendiendo a los niveles de privacidad y seguridad establecidos en la legalidad. La arquitectura del desarrollo compatibiliza con las tecnologías de Moodle, como son PHP y MySQL. De la misma manera, se usa la propia base de datos de Moodle para evitar fugas de datos y centralizarlos en el propio EVA de la institución.

Se resuelve el problema del "listado de la muerte" facilitando encontrar un recurso sin salir del mismo curso o desde cualquier espacio-contexto Moodle.

Se añade que toda interacción con el conector queda recolectada en el $\log$ de Moodle, resolviendo la necesidad de comprensión del uso del chatbot con objetivos de mejora y capacidad analítica de las interacciones de los usuarios.

En términos de trabajos futuros y líneas de investigación, se A pesar de que la interfaz de un chatbot es común en estas tecnologías, como trabajo futuro se deja pendiente de realizar un proceso de experiencia de usuario y usabilidad con los docentes para evaluar la validez de la interfaz del chatbot.

\section{REFERENCIAS}

Amo, D. (2020). Privacidad y gestión de la identidad en procesos de analítica de aprendizaje con Blockchain (Programa de Doctorado Formación en la Sociedad del Conocimiento). Programa de Doctorado Formación en la Sociedad del Conocimiento. Retrieved from https://repositorio.grial.eu/bitstream/grial/1951/1/TesisD anielAmoFilva-LearningAnalytics-Blockchain_v43.pdf

Amo, D., Torres, R., Canaleta, X., Herrero-Martín, J., Rodríguez-Merino, C., \& Fonseca, D. (2020). Seven principles to foster privacy and security in educational tools: Local Educational Data Analytics. ACM International Conference Proceeding Series. https://doi.org/10.1145/3434780.3436637

Amo, Daniel, Alier, M., García-Peñalvo, F. J., Fonseca, D., \& Casañ, M. J. (2020). Protected users: A moodle plugin to improve confidentiality and privacy support through user aliases. Sustainability (Switzerland), 12(6), 2548. https://doi.org/10.3390/su12062548

Ballantyne, E. (2017). Small Optimizations: Five Simple Tweaks to Maximize Student Engagement in Online
Courses. Proceedings of the Atlantic Universities' Teaching Showcase, 21, 33-42.

Catalyst IT. (2017). The First Moodle Chatbot, At Last. MoodleMoot Australia 2017. Retrieved September 1, 2019, from https:/www.lmspulse.com/2017/the-firstmoodle-chatbot-at-last-moodlemoot-australia-2017/

Clarizia, F., Colace, F., Lombardi, M., Pascale, F., \& Santaniello, D. (2018). Chatbot: An education support system for student. Lecture Notes in Computer Science (Including Subseries Lecture Notes in Artificial Intelligence and Lecture Notes in Bioinformatics), 11161 LNCS, 291-302. https://doi.org/10.1007/978-3-03001689-0_23

Dehon, P., Silva, A., Inocêncio, A. C., Castro, C., Costa, H., \& Júnior, P. P. (2018). Cvchatbot: Um chatbot para o aplicativo facebook messenger integrado ao ava moodle. Brazilian Symposium on Computers in Education (Simpósio Brasileiro de Informática Na Educação-SBIE), 29(1), 1623.

Duque, C. T. (2017). Curing The 'Scroll of Death' In Moodle. Retrieved July 9, 2021, from LMSPULSE website: https://www.lmspulse.com/2017/curing-the-scroll-ofdeath-in-moodle/

EP and the CEU. (2016). Regulation (EU) 2016/679 GDPR. Retrieved June 27, 2019, from Official Journal of the European Union website: https://eur-lex.europa.eu/legalcontent/en/TXT/?uri=CELEX\%3A32016R0679

Hien, H. T., Cuong, P. N., Nam, L. N. H., Nhung, H. L. T. K., \& Thang, L. D. (2018). Intelligent assistants in highereducation environments: The FIT-EBOt, a chatbot for administrative and learning support. ACM International Conference Proceeding Series, 69-76. https://doi.org/10.1145/3287921.3287937

La Salle, U. R. L. (2020). MOODLEMOOT 2020 Y GRETEL. Retrieved from https://blogs.salleurl.edu/es/moodlemoot2020-y-gretel

Lande, P. (2016). Avoiding the Scroll of Death - Best Practices - Current Techniques. Teaching with Moodle. Retrieved July 9, 2021, from Moodle website: https://moodle.org/mod/forum/discuss.php?d=336418

Lee, L. K., Fung, Y. C., Pun, Y. W., Wong, K. K., Yu, M. T. Y., \& Wu, N. I. (2020). Using a Multiplatform Chatbot as an Online Tutor in a University Course. Proceedings 2020 International Symposium on Educational Technology, ISET 2020, 53-56. https://doi.org/10.1109/ISET49818.2020.00021

Moodle. (2021). Moodle Statistics. Retrieved June 17, 2021, from https://stats.moodle.org/?lang=es

Oliveira, J. D. S., Espindola, D. B., Barwaldt, R., Ribeiro, L. M. I., \& Pias, M. (2019). IBM Watson Application as FAQ Assistant about Moodle. Proceedings - Frontiers in Education Conference, FIE, 2019-Octob, 1-8. https://doi.org/10.1109/FIE43999.2019.9028667

Pociot, M. (2017). BotMan. Retrieved September 1, 2019, from https://botman.io/

Ranoliya, B. R., Raghuwanshi, N., \& Singh, S. (2017). Chatbot for university related FAQs. 2017 International 
Conference on Advances in Computing, Communications and Informatics, ICACCI 2017, 2017-January, 15251530. https://doi.org/10.1109/ICACCI.2017.8126057

Rovirosa, B., \& Amo, D. (2020). CfM - Chatbot for Moodle. Retrieved from https://github.com/BerniR4/chatbot

Souali, K., Rahmaoui, O., Ouzzif, M., \& El Haddioui, I. (2019). Recommending moodle resources using chatbots. Proceedings - 15th International Conference on Signal Image Technology and Internet Based Systems, SISITS 2019, 677-680. https://doi.org/10.1109/SITIS.2019.00110
SpringML. (2019). Moodle Integration with DialogFlow(chatbots). Retrieved September 1, 2019, from https://springml.com/blog/moodle-integration-withdialogflowchatbots/

Wijaya, E., \& Pebriantara, P. (2018). Rancangan Bangun Aplikasi Pembelajaran dengan Memanfaatkan Chatbot API Dialogflow dan Moodle Berbasis Android Pada SMA IT ALIA Tangerang. @is The Best [Accounting Information System \& Information Technology Business Enterprise], 3(2), 328-335. https://doi.org/10.34010/aisthebest.v3i2.1522 